Review

\title{
Current Approaches and Challenges for Monitoring Treatment Response in Colon and Rectal Cancer
}

\author{
Elizabeth McKeown¹, Daniel W. Nelson², Eric K. Johnson², Justin A. Maykel², Alexander Stojadinovic4, \\ Aviram Nissan ${ }^{5}$, Itzhak Avital ${ }^{6}$, Björn LDM Brücher ${ }^{6}$, Scott R. Steele ${ }^{\circledR}$ \\ 1. Department of Surgery, Swedish Medical Center, Seattle, WA, USA \\ 2. Department of Surgery, Madigan Army Center, Tacoma, WA, USA \\ 3. Division of Colorectal Surgery, UMass Medical Center, Worcester, MA, USA \\ 4. Department of Surgery, Division of Surgical Oncology, Walter Reed National Military Medical Center, Bethesda, MD, USA \\ 5. Department of Surgery, Hadassah-Hebrew University Medical Center, Jerusalem, Israel \\ 6. Bon Secours Cancer Institute, Richmond, VA, USA
}

$\triangle$ Corresponding author: Scott R. Steele, MD, Madigan Army Medical Center, Department of Surgery, 9040a Fitzsimmons Dr., Tacoma, WA 98431. Phone: 253-968-2200; Fax: 253-968-0232; Email: harkersteele@me.com

( ) Ivyspring International Publisher. This is an open-access article distributed under the terms of the Creative Commons License (http://creativecommons.org/ licenses/by-nc-nd/3.0/). Reproduction is permitted for personal, noncommercial use, provided that the article is in whole, unmodified, and properly cited.

Received: 2013.10.01; Accepted: 2013.11.25; Published: 2014.01.01

\begin{abstract}
Introduction: With the advent of multidisciplinary and multimodality approaches to the management of colorectal cancer patients, there is an increasing need to define how we monitor response to novel therapies in these patients. Several factors ranging from the type of therapy used to the intrinsic biology of the tumor play a role in tumor response. All of these can aid in determining the ideal course of treatment, and may fluctuate over time, pending down-staging or progression of disease. Therefore, monitoring how disease responds to therapy requires standardization in order to ultimately optimize patient outcomes. Unfortunately, how best to do this remains a topic of debate among oncologists, pathologists, and colorectal surgeons. There may not be one single best approach. The goal of the present article is to shed some light on current approaches and challenges to monitoring treatment response for colorectal cancer.

Methods: A literature search was conducted utilizing PubMed and the OVID library. Key-word combinations included colorectal cancer metastases, neoadjuvant therapy, rectal cancer, imaging modalities, CEA, down-staging, tumor response, and biomarkers. Directed searches of the embedded references from the primary articles were also performed in selected circumstances.

Results: Pathologic examination of the post-treatment surgical specimen is the gold standard for monitoring response to therapy. Endoscopy is useful for evaluating local recurrence, but not in assessing tumor response outside of the limited information gained by direct examination of intra-lumenal lesions. Imaging is used to monitor tumors throughout the body for response, with $\mathrm{CT}, \mathrm{PET}$, and MRI employed in different circumstances. Overall, each has been validated in the monitoring of patients with colorectal cancer and residual tumors.

Conclusion: Although there is no imaging or serum test to precisely correlate with a tumor's response to chemo- or radiation therapy, these modalities, when used in combination, can aid in allowing clinicians to adjust medical therapy, pursue operative intervention, or (in select cases) identify complete responders. Improvements are needed, however, as advances across multiple modalities could allow appropriate selection of patients for a close surveillance regimen in the absence of operative intervention.
\end{abstract}

Key words: Neoadjuvant therapy, colorectal cancer, treatment response, CEA, imaging modalities, PET, CT, MRI, ERUS, down-staging 


\section{Introduction}

Colorectal cancer represents the second leading cause of cancer-related death in the United States and the third leading cancer overall in men and women, with an age-adjusted incidence rate of 46.3 per 100,000 men and women per year. ${ }^{1}$ It is estimated that over 73,000 men and 70,000 women will be diagnosed with colorectal cancer annually, and that nearly 52,000 people died of colorectal cancer in 2012. Rectal cancer makes up approximately one-third of all colorectal cancers. ${ }^{1}$ Unfortunately, up to $50 \%$ of colon cancer patients will be diagnosed with hepatic metastases at any time in their disease course, ${ }^{2}$ and between $15 \%$ and $25 \%$ have liver metastases at the time of diagnosis. ${ }^{3}$ As such, rectal cancer patients and those with metastatic disease comprise a significant segment of the colorectal cancer population.

Historically, an up-front attempt at curative surgical resection was the cornerstone of management for malignancy of the colon and rectum. ${ }^{4}$ As chemotherapy and radiation therapy have evolved and become more effective, treatment paradigms have diverged, especially for locally advanced mid- to low rectal cancers and metastatic colon lesions. ${ }^{5}$ Furthermore, even in select cases of locally advanced colon cancer, successful R0 resection has been feasible following neoadjuvant chemoradiation therapy (CRT) - something that previously was unattainable. ${ }^{6}$

Yet, despite these improvements, the multidisciplinary team has to be able to accurately identify and differentiate patients who are responding adequately to therapy from those that will require a change in their treatment course. With advancements in preoperative multi-modality therapy, this growing ability to define how we currently measure treatment response on an objective level may also improve our ability to identify "complete responders" that may avoid the morbidity of radical operations altogether. ${ }^{7}$ Clear guidelines have been established by the American Society of Colon and Rectal Surgeons (ASCRS), American Society of Clinical Oncology (ASCO), and National Cancer Care Network (NCCN) for surveillance of colorectal cancer following curative treatment, ${ }^{8}$ but we have no similar evidence-based guidelines regarding the monitoring of treatment response. Questions that still need to be elucidated include: How do we define response - clinically, radiological$1 y$, and pathologically? What are the best means of measuring response to treatment? What is the data behind our currently most-used modalities, including serum testing, imaging, and endoscopy? Can we predict tumor response well enough to completely avoid surgery in appropriate patients? Since optimal management of colon and rectal cancer requires a multidisciplinary approach, effective communication and defined language will also be necessary among specialists. As one example, Kosinski points out there are many different terms, not all of them scientific, describing tumor response, specifically regarding rectal cancer response to neoadjuvant therapy prior to resection. "Down-staging", "downsizing", and "tumor regression" have all been used by pathologists, surgeons, radiologists, and oncologists to describe some very different circumstances. $^{9}$ Multidisciplinary communication is key in providing care to these patients in whom timing of chemoradiotherapy and resection is not always clear-cut.

The purpose of this manuscript is to examine the current approaches and challenges we have in monitoring treatment response for patients with colorectal cancer; specifically, patients with Stages II and III rectal cancer who undergo neoadjuvant chemoradiation therapy (nCRT) and patients with metastatic colorectal cancer. We will discuss current strategies for therapy, define tumor response on a clinical and pathologic level, and review the data regarding modalities for monitoring response (including imaging and serum testing). Finally, we will delve into how we manage those patients who are complete responders on imaging following neoadjuvant chemoradiotherapy.

\section{Current Strategies for Treatment}

\section{Colon cancer therapy}

At present, the treatment for colon cancer remains primarily surgical. Colon cancer is resected either through an open or minimally invasive approach following standard oncological principles that have withstood the test of time.10,11

For Stages I - III colon cancer, resection to R0 status, along with a proper lymphadenectomy, is the gold standard therapy. Stage III and select Stage II patients may also benefit from adjuvant chemotherapy, ${ }^{12}$ although neoadjuvant therapy has little role for localized, resectable colon cancer. ${ }^{11}$ The exceptions to this management occur in those patients presenting with locally advanced near-obstructing colon cancer in which a full evaluation has not been performed, or in those with known metastases that are potentially resectable. ${ }^{11}$ While overall there is a little data on the former cohort, a recent retrospective study was undertaken analyzing 33 patients with potentially resectable, non-metastatic locally advanced adherent colon cancer (i.e., T3-T4) who had received nCRT followed by multi-visceral resection. Of note, an R0 resection was feasible in all patients. These patients were then followed for three years and found to have an overall survival of $85.9 \%$ and disease-free survival of $73.7 \% .{ }^{6}$ Based on this data, it may be feasible and 
beneficial to treat locally advanced adherent colon cancer with neoadjuvant instead of traditional initial primary resection. While definitive recommendations await further experience, this highlights the need to identify those patients with an appropriate response to allow for clear margins.

\section{Metastatic colon cancer therapy}

Treatment for metastatic colorectal cancer primarily consists of systemic chemotherapy with FOLFOX/FOLFIRI (5FU, leucovorin, oxaliplatin versus 5FU, leucovorin, and irinotecan,), all of which have similar responses but different adverse events, ${ }^{11}$ and in select cases, resection of the primary and/or metastatic lesions. When a patient fails to have progression-free survival, the chemotherapy regimen is modified in an attempt to achieve response. ${ }^{11}$ Overall, data on this is mixed, though in general has limited long-term survival. In one study, therapy with leucovorin, irinotecan, oxaliplatin, plus 5-FU were randomly assigned in two-drug combinations to 795 patients with metastatic colon cancer. FOLFOX demonstrated superior results to any of other regimen, with time to progression of 8.7 months, response rate of $45 \%$, and median survival of 19.5 months. ${ }^{13}$ If the tumor develops resistance, other alternatives including anti-EGF or VEGF receptor therapy are commonly instituted. Monoclonal antibody therapy, such as bevacizumab and/or cetuximab, may also be used as an adjunct for treating metastatic disease, either as first-line or as salvage chemotherapy. ${ }^{14}$ When bevacizumab was added to a regimen of 5-FU, irinotecan, and leucovorin, there was a significantly prolonged survival from 15 months to 20 months. ${ }^{15}$

Historically, metastatic colon cancer has been treated exclusively with chemotherapy and biologics, with resection and diversion reserved for those patients that became obstructed, perforated, or bled. At present, there appears to be good data showing that there is improved survival and quality of life with resection of the primary before symptoms, even in the setting of metastatic disease. ${ }^{16-20}$ Highlighting this, five abstracts from a recent ASCO meeting were presented related to the management of the primary tumor. In one of these, the authors performed a retrospective study looking at 517 patients with metastatic colon cancer by way of a propensity score matched analysis that included age, gender, ECOG, and palliative chemotherapy to palliative resection, and found the patients who received palliative resection had overall improved survival (hazard ratio $0.66, p$ value 0.0019). ${ }^{18}$ Another looked at a cohort of 1,378 patients with metastatic colon cancer, demonstrating that resection of the primary tumor was associated with a median overall survival of 18 months versus 8 months
$(\mathrm{P}<0.0001)$ in the non-surgical group. They concluded that resection improves survival independent of chemotherapy, age, functional status, and comorbidities. ${ }^{16}$

Treatment of metastatic colon and rectal cancer also depends on whether the metastases are widespread, involve multiple organs, and are potentially resectable. A consensus statement published in 2005 involving 16 oncologists, surgeons, and radiologists looked at criteria that are used in clinical practice to determine if hepatic metastases are resectable. They found that there was agreement to resect $<4$ metastases, or $>=4$ metastases if involving only one lobe. If $>4$ metastases, or if bilobar involvement, there was a preference to wait until neoadjuvant therapy was able to shrink the tumor. ${ }^{2122}$

\section{Rectal cancer therapy}

Rectal cancer has undergone an evolution of treatment. Initially, Dr. Miles theorized that he could decrease the rates of local recurrence by performing a more extensive lymphadenectomy with his one stage abdominoperineal resection of the rectum and sphincter complex, leaving the patient with a permanent end colostomy. ${ }^{23}$ Over the course of time, with advances in stapler technology, operative technique such as a total mesorectal excision (TME) and a better understanding of the margin necessary to ensure a better balance between oncologic outcome and functional outcomes, distal cancers of the rectum have been increasingly treated with sphincter-sparing surgery. ${ }^{4}$ Multiple reports have validated the use of neoadjuvant chemoradiation in the treatment of AJCC Stage II and III rectal cancers, as well as use of chemotherapy for both palliation and cure in lymph node-positive and metastatic colon cancer. ${ }^{24-35}$ Interestingly based on our current stratagem for treating rectal cancer, a randomized control trial performed in 1984 examined 824 patients, grouping them into three arms: surgery alone, preoperative $2000 \mathrm{rad}$ in 10 daily fractions, and preoperative $500 \mathrm{rad}$ as a single fraction. They found no difference in the 5-year actuarial survival, with similar local control, distant metastases, and complication rates. ${ }^{27}$ Allal examined a retrospective cohort of 53 patients with rectal cancers within 6 $\mathrm{cm}$ of the anal verge who received neoadjuvant radiotherapy with or without 5-FU. Sphincter-sparing surgery was performed in 23 of those patients, and an APR was performed in 29 patients, with no difference in loco-regional control or overall survival. ${ }^{24}$ In a landmark trial, Sauer and colleagues randomized 421 rectal cancer patients to preoperative versus post-operative chemoradiotherapy along with total mesorectal excision. While survival rates were similar (76\% preoperative vs. $74 \%$ post-operative), the 
five-year local recurrence rate was significantly improved in the nCRT group ( $6 \%$ vs. $13 \%, \mathrm{P}<0.01)$. Additionally, a decreased rate of acute toxic effects from the chemoradiotherapy was noted in those patients treated before surgery. ${ }^{33}$

Based on these and more recent studies, nCRT provides not only an improved local control and sphincter-sparing ability, but also similar quality of life and overall survival, making it the preferred option for locally advance rectal cancer lesions.

\section{Surveillance after complete resection}

Surgical resection of a colon cancer involves standard oncological principles with at least five centimeter proximal and distal margins, negative circumferential margins, and removal of a minimum of twelve lymph nodes. This is considered a complete (R0) resection, ${ }^{11}$ and post-operative surveillance is undertaken according to NCCN, ASCRS, and ASCO recommendations.

\section{Defining Tumor Response}

The terms used to define the response of a tumor have evolved to reflect the modalities that are in use. Proper patient selection and optimizing outcomes require clinicians to integrate all of these terms in order to form a cohesive picture of tumor location, size, is and how it has changed over time. Just as an autopsy often provides the definitive answer to a mortal disease process, the pathologic description of a resected tumor gives us the best data for that lesion at that moment in time. Clinical descriptors such as fixed, relationship to the sphincter complex, associated lymphadenopathy, and suspected invasion into adjacent structures - that is, non-pathologic predictive descriptors of a tumor's response to therapy obtained from office tests, serum tests, and imaging - are used as a surrogate for pathologic definitions.

Some descriptors are used indiscriminately with regard to a tumor's response after therapy. Technically speaking, "down-staging" should be used to describe a tumor's decline from one AJCC Stage to another, though is often used incorrectly when size alone changes. To avoid this, Kosinski proposes a shift of terminology from "down-staging" to "downshifting", to reflect less dramatic changes in individual $\mathrm{T}$, $\mathrm{N}$, or M classifications. Her group also advocates using "tumor regression" only as it refers to pathologic changes. ${ }^{9}$ In this section we will discuss modalities currently used to define a tumor's response to treatment.

\section{Clinical evaluation}

\section{Digital Rectal Exam (DRE)}

Digital rectal examination is the classic assess- ment of a rectal lesion by palpation. It is routinely used to clinically gauge the size of the lesion, presence of ulceration, location of the lesion relative to the anal verge, functional status of the sphincter complex, and percentage of the lumen occluded. For low-lying lesions that are palpable, it provides the additional advantage of easy repetition and the ability to follow these aspects over time and after therapy. DRE also has value in determining the presence or absence of a fixed or tethered state as a surrogate for AJCC T-stage, and gives a rough estimate of circumferential resection margin involvement. Out of 245 patients who underwent surgery initially, assessment of circumferential resection margin was predicted more accurately with MRI versus DRE (PPV, 92\% versus 70\%) in the same group of patients. ${ }^{36}$ Another study evaluated DRE as a cost-effective initial staging test, comparing it against MRI and endorectal ultrasound (ERUS) as compared to judging favorability, meaning direct extension into mesorectal fat or node positivity, to determine whether or not nCRT would be beneficial for the patient. Unfortunately, there was only a $65 \%$ agreement rate between staging by DRE and histological assessment of tumor favorability. ERUS performed slightly better, at $69 \%$ agreement between preoperative assessment and pathologic assessment, and MRI had a $94 \%$ assessment of favorability as correlated to pathology. ${ }^{37}$ Another study showed that DRE underestimated the extent of rectal cancer response after nCRT in $78 \%$ of patients, thus severely limiting its utility in this role. ${ }^{38}$ Despite its limitations, DRE is a risk-free, simple test that can be performed repeatedly to gauge a rough estimation of tumor response.

\section{Carcinoembryonic antigen (CEA)}

Gold and Freedman first described carcinoembryonic antigen of the human digestive system in 1965. It is currently the most widely utilized tumor marker for colon cancer. A serum glycoprotein, CEA is useful as a surrogate for disease recurrence in tumors that secrete it. ${ }^{39}$ In colorectal cancer, it has a sensitivity and specificity of $36 \%$ and $87 \%$ in screening for early colon cancer. ${ }^{40}$ Its most useful role in surveillance is after $\mathrm{R} 0$ resection in colon cancer, but has limitations as a marker of recurrence - as transient elevations of CEA can be seen in times of active inflammation of the colon, such as a flare of ulcerative colitis, after some chemotherapies, and in benign liver disease. ${ }^{41}$ It can also be mildly elevated in tobacco smokers. Overall, there is a false positive rate of 7 to $16 \% .42$

\section{Rectal Cancer}

Obtaining a serum CEA to monitor response 
following nCRT in rectal cancer has no consensus among surgeons or oncologists. CEA has demonstrated some utility when values decrease after neoadjuvant chemoradiotherapy and are followed, indicating a good prognosis and has been correlated with tumor response and complete tumor regression. Perez et al looked at 170 patients undergoing nCRT and found a post-treatment level of $<5 \mathrm{ng} / \mathrm{ml}$ was associated with increased rates of complete clinical and pathologic response, as well as increased overall $(P=0.01)$ and disease-free survival $(p=0.03)$. Of note, there was no correlation between initial CEA or the degree of reduction in CEA with tumor response or overall survival. ${ }^{43}$

\section{Metastatic Colon Cancer}

In terms of monitoring response during the treatment for metastatic disease, CEA should be measured prior to the start of therapy and every two to three months thereafter, with elevations of around $30 \%$ from the patient's baseline associated with a poor response to therapy. ${ }^{42}$

In sum, while CEA is an excellent test when used in conjunction with imaging modalities for determining recurrence, it can also be used in selective circumstances as a marker of prognosis and tumor response for rectal cancer patients who have initially high values that decline after nCRT, as well as those patients undergoing systemic therapy for metastatic colon cancer.

\section{CEA-Derived Tests}

Tumor localization by polyclonal antibodies against CEA in xenografts of colon cancer was first described in 1973. It has had a small following of believers that routinely use it in this capacity since then. Studies came out in 2000 and 2001 comparing CEA scan and FDG-PET in this same patient population, showing that CEA scan had a sensitivity of $18 \%$, a specificity of $33 \%$, a positive predictive value of $50 \%$, and a negative predictive value of $10 \%$. Unfortunately, it also failed to predict resectability in any patient. ${ }^{44,45}$

Radiolabelled isotope-guided surgery (RIGS) showed initial promise and was utilized mostly in the 1990s. Using intraoperative quantification of cells after injection of monoclonal antibodies, this technique used a hand-held scanner to detect high concentration of cells producing CEA. In one study, occult tumor was detected in 30 sites in 26 patients, and changed management in $1 / 3$ of patients with recurrent excisions. ${ }^{46}$ In mouse studies, the sensitivity and specificity of RIGS for tumor localization is $71.4 \%$ and $91.4 \%$, respectively ${ }^{47}$ In the absence of any randomized trials or newer data, its use has tapered over the last decade, and likely has a limited role (if any) in determining treatment response.

\section{Other Markers}

There are currently other serum tests that have potential value for surveillance, prognosis, and targeted therapy; however, none are currently available or applicable for practical use in monitoring treatment response. DNA methylation, isolated by PCR from DNA samples from blood or stool, ${ }^{48}$ has the potential to be useful in proximal colon cancer but not rectal cancer. ${ }^{49}$ In a review of 161 patients, cells were evaluated to determine the presence of CIMP (CpG Island Methylator Phenotype). This mutation was found to be an independent prognostic factor for disease-free survival in Stage 3 proximal colon cancer, but has limited role at present in following treatment response. ${ }^{49} \triangle \mathrm{TAp} 73$ is another marker in colon cancer that has been associated with shortened overall survival, increase in proliferation, and drug resistance in small series. ${ }^{50}$ Circulating tumor cells, detected by CEA mRNA as well as human telomerase reverse transcriptase, cytokeratin-19, and cytokeratin-20, also have wonderful potential as markers of recurrence, but again, no current utility as markers of tumor response. ${ }^{51} \mathrm{Lu}$ et al performed analysis on 141 patients and found that presence of persistent postoperative CTCs was correlated with postoperative early relapse in locally advanced colorectal cancers. ${ }^{52}$ Thymidylate synthase is another marker for prognosis - a meta-analysis published in 2004 examined twenty studies identifying overall prognosis and progression free survival in colorectal cancer patients with thymidylate synthase (TS) levels, and concluded that tumors expressing high levels of TS appeared to have a poorer overall survival compared with those expressing low levels. ${ }^{53}$ Overall, these markers all have potential use in the future for prognosis, and can easily speculate how they may eventually find a role in monitoring response, but unfortunately have no current role in determining response of the primary tumor or metastases to surgical or chemoradiotherapy.

\section{Imaging}

The use of imaging in the staging and recurrence of colorectal cancers has undergone evolution and improvement over the last twenty years. There is critical importance in initially staging rectal cancers and determining lymph node involvement, as this will determine the need for neoadjuvant CRT. In general, accuracies for imaging for rectal cancer for initial staging are variable and often user-dependent. Bipat et al performed a meta-analysis of ninety articles attempting to answer the question of which modality provided the best information for local staging and 
lymph node involvement in the initial assessment of rectal cancer. They found that ERUS and MRI had similar sensitivities for muscularis propria invasion, but that ERUS was significantly more specific than MR ( $86 \%$ versus $69 \%$, p value of 0.02 ), and the authors concluded from this that MRI overstaged T1 tumors. Ultrasound was also significantly more sensitive $(90 \%$ versus $79 \%$ and $82 \%$, respectively) for perirectal tissue invasion ( $p$ 0.003) than either CT or MRI. ${ }^{54}$

Though there is good data to suggest ideal timing for resection after preoperative therapy for rectal cancer, ${ }^{55-58}$ there is no consensus about the need for, or even timing of imaging after completion of neoadjuvant therapy. In general, a majority of surgeons advocate resection based on pre-radiotherapy tumor status, disregarding potential post-nCRT downstaging.

A question is advanced about how to manage those patients who have big changes after neoadjuvant therapy - do they still require radical surgery? The theory of a "wait and see" policy, which avoids surgical resection in favor of closely following those patients who have had a clinical complete response after neoadjuvant therapy for rectal cancer, originated after an initial observational study in Brazil by Habr-Gama. In a study of 260 patients with low rectal cancer deemed resectable, all patients underwent nCRT, with those experiencing an incomplete response taken for resection. Those patients that had a complete clinical response were followed closely under a strict protocol governed by routine clinical examination, laboratory testing, and imaging. Overall survival rates were significantly higher in the clinical complete responders compared with those who underwent resection and were found to be pathological complete responders $(p=0.01)$. Disease free survival was also higher in clinical complete responders. ${ }^{59}$

Imaging modalities are notoriously poor at predicting lymph node involvement after radiation, ${ }^{60,61}$ and Brown et al demonstrated that among 5 millimeter lymph nodes, malignancy could be harbored in as many as $28 \%$ of rectal cancer patients. ${ }^{62}$ Though there are standardized schemas to grade tumor response by imaging, there are no studies examining how treatment can be guided by imaging alone.

There have also been a number of studies attempting to establish protocols for the best monitoring modalities after nCRT (Table 1), in part to better judge the clinical complete responders, but also to monitor the tumor in-situ and best determine operative approach. There are clearly individuals that respond completely to neoadjuvant therapy, and a clinical complete response has been shown by some to correlate well with a pathologic complete response. However, questions have arisen regarding the ability of imaging to predict these patients and who can, potentially, avoid resection.

\section{Computed Tomography (CT)}

The accuracy of CT in evaluating rectal cancer is limited by its ability to distinguish layers of the bowel wall, ${ }^{63}$ and studies have shown that CT is not an accurate modality for determining the degree of pathologic response. ${ }^{64}$ However, CT scans are both sensitive and specific for the detection of metastatic disease, and are considered the gold standard for determining the presence or absence of metastatic colorectal cancer in particular. ${ }^{65}$ Scans are both costly and come with an undeniable radiation risk (especially for younger patients who may survive for dozens of years).

On the other hand, CT scans have much more value in monitoring metastatic disease because they are excellent in determining tumor size and visceral involvement, but do not allow further characterization of tumor heterogeneity and evolution over time. ${ }^{66} \mathrm{CT}$ measurements are operator-dependent, and there have been discrepancies among experienced physicians from $15-40 \%{ }^{67}$ Tumor response using CT scans is measured by RECIST (Response Evaluation Criteria in Solid Tumors) criteria, which looks at the longest dimension of a lesion and grades response according to a partial response (PR), progressive disease (PD), or stable disease (SD). ${ }^{65}$ While CT has a number of limitations, its high sensitivity in determining the size of lesions provides for an excellent option to monitor effects of adjuvant therapy on metastatic disease, in addition to helping determine progression-free survival.65,66

\section{Positron Emission Tomography (PET)}

PET has a higher sensitivity and specificity than CT and is recommended for use in a metastatic workup for colorectal cancer in the setting of an elevated CEA or when there is concern for distant metastases. Huebner et al performed a meta-analysis of the literature for FDG-PET in the detection of recurrent colorectal cancer, reporting that it had an overall sensitivity of $97 \%$ and a specificity of $76 \% .68$ PET parameters include evaluating volume, largest diameter, and mean SUV. Limitations include missing small $(<1$ $\mathrm{cm}$ ) lesions, poor spatial resolution, and a high false positive rate for uninvolved lymph nodes. A systematic review published in 2011 found insufficient evidence to support its routine use in the workup of primary colorectal cancer, ${ }^{69,70}$ and the national guidelines do not recommend routine use of PET in the workup of non-metastatic colorectal cancer. ${ }^{71}$

Unlike CT scans, there is no updated, definitive classification scheme for evaluating tumor response in PET scans. The EORTC (European Organization for 
Research and Treatment of Cancer PET study Group), published in 1999, ${ }^{65}$ looked at SUV within the tumor region as compared to the baseline scan, and graded this as progressive metabolic disease (PMD), stable metabolic disease (SMD), partial metabolic response (PMR), or complete metabolic response (CMD). Many studies have evaluated PET as a restaging agent, and have found a sensitivity of $95 \%$, specificity of $98 \%$, and accuracy of $98 \% .72-76$ A RCT evaluated PET CT in restaging stages II and III rectal cancer, and found that it had a sensitivity of $45 \%$, specificity of $79 \%$ and an overall accuracy of staging and disease response of $56 \%$ as compared to pathologic response. ${ }^{77}$ Based on that study PET is poor marker of clinical response.

To date, PET-CT following chemoradiation has not been able to predict the pathologic response in locally advanced rectal cancer. ${ }^{78}$ Some evidence sup- ports the accuracy of PET in predicting pathologic complete response, ${ }^{79}$ while other evidence disputes its utility. ${ }^{64}$ In a prospective study by Guillem et al, 121 patients with rectal cancer underwent PET and CT before and after neoadjuvant chemoradiation. The results of these imaging studies were compared to pathologic specimens in an attempt to investigate the accuracy of both modalities in predicting a complete pathologic response. Both modalities were inadequate at distinguishing a pathologic complete response from an incomplete one. PET and CT performed poorly at detecting pathologic complete responders (54\% and 19\% were detected by PET and CT, respectively), though they were good modalities for detecting incomplete responders, with $66 \%$ and $95 \%$ on PET and CT, respectively. ${ }^{64}$

Table I. Comparison of Imaging Modalities for Evaluation of Response of Rectal Cancer Following Neoadjuvant CRT.

\begin{tabular}{|c|c|c|c|c|}
\hline Author/Year & Modality & Protocol & Data & Conclusions \\
\hline Chennupati, $2012^{111}$ & PET & $\begin{array}{l}\text { All patients who received both } \\
\text { pre- and post-CRT PET scans. } \\
\text { Compared PET results to TRG }\end{array}$ & $\begin{array}{l}35 \text { patients; } \\
\text { No correlation between SUV, met- } \\
\text { abolic tumor volume between path- } \\
\text { ologic responders versus } \\
\text { non-responders }\end{array}$ & $\begin{array}{l}\text { Changes on PET have } \\
\text { limited value in pre- } \\
\text { dicting pathologic } \\
\text { response of rectal } \\
\text { cancer after neoadju- } \\
\text { vant CRT }\end{array}$ \\
\hline MERCURY, $2007^{112}$ & MRI & $\begin{array}{l}\text { MRI preoperatively at an average } \\
\text { of } 25 \text { days before surgery. Only } \\
\text { short duration radiotherapy in- } \\
\text { cluded. }\end{array}$ & $\begin{array}{l}679 \text { patients with rectal cancer; } \\
\text { MRI vs. pathological examination of } \\
\text { extramural depth }\end{array}$ & $\begin{array}{l}\text { MRI and histopatho- } \\
\text { logic assessment of } \\
\text { tumor spread corre- } \\
\text { lated within } 0.5 \mathrm{~mm}\end{array}$ \\
\hline Brown, $2004^{37}$ & $\begin{array}{l}\text { ERUS, DRE, } \\
\text { MRI }\end{array}$ & $\begin{array}{l}\text { Each of three modalities per- } \\
\text { formed at baseline and repeated } \\
\text { two weeks prior to surgery. Both } \\
\text { early stage rectal cancers and those } \\
\text { receiving nCRT included. } \\
\text { Compared each to final pathology. } \\
\text { Assessed favorability (invasion, } \\
\text { nodal involvement) }\end{array}$ & $\begin{array}{l}98 \text { patients undergoing TME; } \\
\text { MRI } 94 \% \text { agreement with pathology; } \\
\text { DRE } 65 \% \text { agreement with patholo- } \\
\text { gy; } \\
\text { ERUS } 69 \% \text { agreement with pathol- } \\
\text { ogy; }\end{array}$ & $\begin{array}{l}\text { MRI is a better pre- } \\
\text { dictor of tumor re- } \\
\text { sponse }\end{array}$ \\
\hline $\begin{array}{l}\text { Wieder, Geinitz, } \\
2007^{113}\end{array}$ & PET-FLT & $\begin{array}{l}\text { PET prior to CRT, two weeks after } \\
\text { initiation of CRT, \& 3-4 weeks after } \\
\text { chemotherapy but before resection }\end{array}$ & $\begin{array}{l}10 \text { patients; } \\
\text { Poor correlation with pathologic } \\
\text { specimen. }\end{array}$ & $\begin{array}{l}\text { PET uptake of FLT } \\
\text { decreased steadily. } \\
\text { Did not correlate to } \\
\text { tumor regression. }\end{array}$ \\
\hline Pastor C, $2011^{89}$ & ERUS & $\begin{array}{l}4-6 \text { weeks after neoadjuvant CRT; } \\
\text { Goal of the study was to validate } \\
\text { ERUS as a predictor pathologic } \\
\text { response. } \\
\text { Correlated to pathologic specimen }\end{array}$ & $\begin{array}{l}235 \text { patients; } \\
20 \% \text { misclassified as uN0; } \\
75 \% \text { correct regarding LNs; } \\
\text { Overall, over-staging in } 37 \%\end{array}$ & $\begin{array}{l}\text { ERUS is not accurate } \\
\text { in identification of } \\
\text { positive nodes. }\end{array}$ \\
\hline Denecke, $2005^{79}$ & $\begin{array}{l}\text { MRI, CT, } \\
\text { and PET }\end{array}$ & $\begin{array}{l}\text { Each patient received one of the } \\
\text { modalities before neoadjuvant } \\
\text { CRT and 2-4 weeks after neoadju- } \\
\text { vant CRT. } \\
\text { Compared with ERUS and pa- } \\
\text { thology. }\end{array}$ & $\begin{array}{l}23 \text { patients with T3 or } 4 \text { rectal cancer } \\
\text { after CRT; . FDG PET: } 100 \% \text { (sens), } \\
60 \% \text { (spec); } \\
\text { CT } 54 \% \text { (sens), } 80 \% \text { (spec); } \\
\text { MR } 71 \% \text { (sens), } 67 \% \text { (spec) }\end{array}$ & $\begin{array}{l}\text { PET is superior to CT } \\
\text { and MRI predicting } \\
\text { response to CRT }\end{array}$ \\
\hline
\end{tabular}

Legend: PET: Positronic Emission Tomography; CRT: Chemoradiotherapy; SUV: Standardized uptake value; TRG: Tumor regression grade; MRI: Magnetic Resonance; Imaging;

ERUS: Endoscopic rectal ultrasound; DRE: Digital rectal exam; TME: Total mesorectal excision; PET-FLT: Fluoro-L-Thymidine; LN: Lymph node; CT: Computed omography;

FDG: PET with fludeoxyglucose;

Sens: sensitivity; Spec: specificity. 


\section{Magnetic Resonance Imaging (MRI)}

MRI has been validated as the modality of choice for staging rectal cancer, both before surgery or following neoadjuvant therapy. ${ }^{80,81}$ MRI allows preoperative identification of wall invasion, node classification, depth of spread, venous invasion, possible involved circumferential resection margin, and serosal involvement at or above the peritoneal reflection. ${ }^{82}$

There have been many studies evaluating the use of MRI in the initial staging of rectal cancer. The MERCURY (Magnetic Resonance Imaging and Rectal Cancer European Equivalence Study) ${ }^{36,83}$ trial reported that MRI can accurately predict surgical resection margin and depth of tumor invasion, but did not draw conclusions in the issue of neoadjuvant therapy and its effect on the sensitivity of MRI. A study from Kim and colleagues ${ }^{84}$ utilized a prospective cohort to examine patients with biopsy confirmed rectal cancer, comparing volumetric analysis with MRI, histopathologic down-staging, and tumor regression grade in evaluating tumor response after nCRT. In his study, 30 patients with locally advanced rectal cancer underwent MRI evaluation before and after chemoradiotherapy, and imaging results were compared to the tumor regression grade after surgical resection. This study determined that tumor volume reduction by magnetic resonance volumetry does not correlate with histopathologic down-staging and tumor regression grade. ${ }^{84}$ Another study found validity in MR volumetric studies. In this cohort, 84 patients were evaluated prospectively with volumetrics and percent decrease ratio, and found that $75 \%$ of the tumor volume reduction ratios correlated with a pathologic complete response 85

Quantitative assessment of tissue changes after neoadjuvant therapy for rectal cancer on T2-weighted signal intensity has been shown to potentially identify pathologic complete responders, perhaps identifying those patients who would benefit from the "watch and wait' strategy. A study examined 39 patients with locally advanced adenocarcinoma of the rectum who were imaged with MRI before and after nCRT. Compared to tumor regression grade as the gold standard, MRI had an accuracy of about $92 \%$ for complete response. ${ }^{86}$ In a separate study, MRI was compared with colonoscopy and repeat biopsy of the original lesion. The investigators found that the prediction rate for pathologic complete response was only 33\% with MRI. ${ }^{87}$ The variation in results between studies suggests that inter-observer variation may be a problem.

In summary, MRI is a valuable tool to assess for clinical and pathologic response, but, because it is so specific for soft tissue invasion, it is less accurate after radiation.

\section{Endorectal Ultrasound (ERUS)}

ERUS is a very helpful tool for initial staging of rectal cancer because of its ability to distinguish between histological layers of bowel wall. Its sensitivity and specificity decrease with increasing depth of the rectal lesion, ${ }^{79}$ and its poor assessment of lymph node status after nCRT makes it an unreliable resource for single-modality treatment monitoring ${ }^{88-90}$ In evaluation of rectal lesions before neoadjuvant therapy a group compared MRI and ERUS and found them to have similar accuracies for T stage. For T2 and T3 lesions, ERUS had a sensitivity of $76 \%$. The study found that ERUS understaged all T4 tumors, and that ERUS performed poorly with stenotic lesions and those that were polypoid. ${ }^{91}$ Others have found that ERUS is unreliable for $\mathrm{T}$ stage monitoring after $\mathrm{nCRT},{ }^{92}$ with over-staging being the most common cause of inaccuracy. ${ }^{93}$ Some clinicians believe that ERUS is a valuable adjunct even after nCRT, citing its ability to distinguish maximum depth of the fibrosis associated with the lesion as well as involvement of the sphincters. ${ }^{94,95}$ In a group of twenty-nine patients, preoperative ERUS after nCRT was compared to the pathologic specimen. The authors, found that ERUS predicted the fibrosis that replaced the tumor to an accurate depth. ${ }^{95}$ The major flaw in the use of ERUS after nCRT is its inability to distinguish tumor from fibrosis. It is therefore difficult to make treatment recommendations based on these results, and it is difficult to classify a patient as a clinical complete responder based on this modality.

In addition to its inability to evaluate lymph nodes, and its poor capability to differentiate fibrosis from cancer, ERUS cannot adequately visualize the mesorectal fascia, and so cannot predict the circumferential resection margin at resection. This is yet another reason that ERUS is a poor tool for post-neoadjuvant decision making and operative planning. ${ }^{90,96}$

\section{Endoscopy}

Monitoring for intralumenal recurrence with colonoscopy, flexible or rigid sigmoidoscopy is consistently recommended by governing organizations, albeit at varying intervals. ${ }^{8}$ Endoscopy is useful as an adjunct in detecting intralumenal recurrence, for which it is both highly sensitive and specific. Preoperatively, the value of rigid sigmoidoscopy in rectal cancer cannot be overestimated, as it provides the ability to determine mobility of the mass, appropriate assessment of the distance from the verge to the lesion, and sizing information obtained are invaluable to operative planning. It is also a therapeutic intervention, with ability to biopsy and to excise small lesions, not to mention its value in following the tu- 
mor's response to nCRT, surveying the anastomosis and detecting anastomotic recurrences. However, it carries with it both cost and a small risk to the patient.

Endoscopy has value in monitoring treatment response because of its ability to assess remaining macroscopic tumor, but, as it is unable to determine nodal status and subtle changes or occult spread after therapy, it has no utility as a monomodal therapy .80

\section{Clinical Complete Responders}

No discussion about monitoring treatment response of rectal cancer following nCRT would be complete without examination of the clinical conundrum of what to do with those who appear to have a clinical complete response. This can be seen in $\sim 20-50 \%$ of those patients with rectal cancer undergoing neoadjuvant therapy. ${ }^{97}$ While there is no consensus on what imaging is best to evaluate a clinical complete response (Table 2), when imaging suggests a complete responder, the question arises of whether to proceed with resection of these patients.

Clinical complete response does not always correlate with pathologic response-in fact this occurs less often than previously thought. Hiotis found that of those clinical complete responders, only $25 \%$ of them had a pathologic complete response after resection (though, notably, the method that was used for determining CCR was proctoscopy). ${ }^{97}$ One review examined 38 trials presenting data on those patients who were clinical complete or pathologic responders, and found that T3 cancers that were managed non-operatively were associated with high local recurrence rates..$^{98}$

The challenge comes in knowing which of these patients will benefit from a non-operative approach, and which will not. A prospective cohort study was assembled with the goal of evaluating the safety of a 'wait and see' policy based on MRI, endoscopy, and stringent follow-up. At a mean follow-up of 25 months +/- 19 months, the authors showed that, compared to twenty patients who had a pathologic complete response after resection, disease-free survival and overall survival compared favorably in the 21 patients in the non-operative group with only one patient developing local recurrence and requiring salvage surgery. ${ }^{99}$ Another cohort study looked at 32 patients who were treated non-operatively after a clinical complete response following nCRT. These individuals were compared with a group that had pathologic complete response after resection. Eighty percent of the clinical complete response group avoided resection of the initial tumor, with the six patients who experienced local recurrence undergoing salvage rectal resection. After resection, those six patients had no further local recurrence at a follow up of 17 months. 100

Table 2. Modality as an Indicator of Clinical Complete Response

\begin{tabular}{|c|c|c|c|}
\hline Author/Year & Modality & Results & Conclusions \\
\hline Hiotis, $2002^{97}$ & $\begin{array}{l}\text { DRE and Proc- } \\
\text { toscopy }\end{array}$ & $\begin{array}{l}488 \text { patients; } \\
19 \% \text { CCR } \\
\text { pCR } 10 \% \\
\text { PCR among CCR: } 25 \% \text { agreement }\end{array}$ & $\begin{array}{l}\text { These modalities are not accurate in judging } \\
\text { CCR. }\end{array}$ \\
\hline Guillem, $2005^{38}$ & DRE & $\begin{array}{l}94 \text { patients; } \\
21 \% \text { agreement between pathologic and DRE } \\
\text { assessment of complete response. }\end{array}$ & DRE is not accurate in judging CCR. \\
\hline $\begin{array}{l}\text { Habr-Gama, } 2004 \\
114\end{array}$ & $\begin{array}{l}\text { DRE } \\
\text { Proctoscopy } \\
\text { Colonoscopy } \\
\text { CT } \\
\text { CEA }\end{array}$ & $\begin{array}{l}71 \text { patients ( } 26 \% \text { ) had CCR on combination of all } \\
\text { imaging studies and managed nonoperatively. } \\
\text { Overall 5-year DFS } 92 \% \text {, OS } 100 \% \text { in } \\
\text { non-operative arm compared to DFS } 83 \% \text {, OS } \\
88 \% \text { in those patients who underwent resection. }\end{array}$ & $\begin{array}{l}\text { Non-operative therapy after full assessment of } \\
\text { response is feasible and safe in those patients } \\
\text { who have a clinical complete response and } \\
\text { who can undergo regular surveillance }\end{array}$ \\
\hline Maretto, $2007^{115}$ & $\begin{array}{l}\text { ERUS } \\
\text { CT } \\
\text { MRI }\end{array}$ & $\begin{array}{l}45 \text { patients; } \\
\text { Underwent all modalities after nCRT with com- } \\
\text { parison to pathologic status: } \\
\text { Accuracy for T stage: ERUS } 64 \% \\
\text { CT } 74 \% \\
\text { MRI } 77 \% \text {; } \\
\text { Nodal status for all modalities }<70 \% \text {. }\end{array}$ & $\begin{array}{l}\text { Not predictable agreement between complete } \\
\text { responders and pathologic responders, all } \\
\text { current rectal cancer restarting techniques } \\
\text { give poor accuracy of T-stage. }\end{array}$ \\
\hline Intven $\mathrm{M}, 2013^{116}$ & MRI & $\begin{array}{l}59 \text { patients with locally advanced rectal cancer; } \\
22 \% \text { were 'Good Responders (GR)'; } \\
\text { Positive predictive value of MRI for pCR and GR } \\
\text { was } 64 \% \text { and } 91 \% \text {, respectively. }\end{array}$ & $\begin{array}{l}\text { MRI can predict good responders fairly accu- } \\
\text { rately, but has a poor predictive value for } \\
\text { pathologic complete responders }\end{array}$ \\
\hline
\end{tabular}

Legend: PCR: Pathologic complete response; CCR: Complete clinical response; CEA: Carcinoembryonic antigen; DFS: Disease free survival; OS: Overall survival; CRT: Chemoradiotherapy; SUV: Standardized uptake value; TRG: Tumor regression grade; MRI: Magnetic Resonance Imaging; ERUS: Endoscopic rectal ultrasound; DRE: Digital rectal exam; TME: Total mesorectal excision; PET-FLT: Fluoro-L-Thymidine; LN: Lymph node; CT: Computed tomography; FDG: PET with fludeoxyglucose. 


\section{Pathologic evaluation}

Pathologic evaluation is the gold standard for defining primary tumor response, especially in assessing rectal cancer patients after neoadjuvant chemoradiotherapy and resection. Pathologic evaluation reports accurate information on presence of residual tumor, lymph node status, and tumor response to therapy. Non-neoplastic portions of the surgical specimen can demonstrate confounding pathologic changes after nCRT, just as post-radiation radiographic changes are seen on ERUS. Aside from tumor regression, ulceration, submucosal fibrosis, mucin lakes, and calcification ${ }^{101}$ can be seen. All of these factors make evaluation of the primary tumor challenging.

The need for standardization of pathologic response reporting has driven the development of a grading system for pathologists. Tumor regression grade (TRG), first described in esophageal surgery following neoadjuvant therapy as the tumor mass compared to the converted scar in a ratio, ${ }^{102}$ is an independent predictor of survival in rectal cancer. Those showing pathologic complete response (pCR) have improved disease free survival, decreased local recurrence, and increased overall survival (OS). TRG 0 is classified as a complete response, TRG 1 is a moderate response, TRG 2 is a minimal response, and TRG 3 is no response. ${ }^{103}$ In a study of 108 patients who received neoadjuvant CRT for rectal cancer, it was found that those with a total regression (TRG 0) had an improved three-year disease-free survival over those with less regression. ${ }^{104}$ Rodel et al evaluated TRG in 385 patients treated with preoperative chemoradiotherapy, using an inverse scale as the one above; that is, they classified TRG 4 as no tumor and TRG 0 as complete absence of fibrosis. They revealed that five-year DFS after CRT and curative resection was $86 \%$ in their tumor-free arm and 63\% in their TRG 0 group. On multivariate analysis of their data, they determined the pathologic T-stage and nodal status upon restaging to be the most important independent prognostic factors, but that decreasing tumor to fibrosis ratios suggested improved DFS. 105 Tumor regression grade, which has been shown to be a good surrogate for disease free survival, gives surgeons an objective way to evaluate tumor response to therapy.

Tumor regression grade zero, or complete absence of tumor following nCRT, is associated with very low rates of local recurrence and excellent long-term survival. A systematic review and meta-analysis of 16 studies involving over three thousand patients examined individuals who had rectal cancer and received nCRT. Following interval proctectomy 1263 were found to have complete re- gression of their tumor. ${ }^{106}$ Distant failure was observed in $9 \%$, local recurrence rate was $0.7 \%$, and overall and disease free-survival rates were $90 \%$ and $87 \% .{ }^{106}$ Another study performed pooled analysis of twenty-seven articles in which patients underwent nCRT and TME. Kaplan Meier survival graphs were calculated and subgroup analyses were performed to look for confounding factors. Out of 3,100 patients, 484 had a pathologic complete response $(16 \%)$. Five year disease-free survival was $83.3 \%$ for those patients, and $65 \%$ for those patients without $\mathrm{pCR}$. This effect was not confounded by other variables. ${ }^{107}$

Comparison of pretreatment and pathologic staging has revealed that depth of invasion is significantly downshifted after nCRT ${ }^{108}$, which also correlates with decreased local recurrence and increased sphincter preservation, though there is no difference in survival. ${ }^{33}$ Fifty-seven patients were investigated who underwent neoadjuvant radiotherapy or chemoradiotherapy in 2004 who then underwent resection with TME. Of the specimens obtained, 21\% had a "good" response, $44 \%$ had a "median" response, and 35\% had a "poor" response. They found $65 \%$ of patients had a significant tumor regression, meaning either median or good response. Ten percent had complete pathologic response and $10 \%$ had only microscopic foci of adenocarcinoma. ${ }^{108}$

A study was performed to determine if nodal status could be successfully predicted by $\mathrm{pCR}$ in the primary specimen after nCRT, which may allow for local excision of the primary site alone of higher-grade rectal cancers with a low risk of pelvic recurrence. Over 140 patients with T3 or T4 rectal cancers were examined. Eighteen percent of these were noted to have had complete tumor regression after nCRT, with four out of the eighteen patients (17\%) of this selection having involved lymph nodes. The degree of pathologic response did correlate with a lymph node response, possibly allowing local excision in the future of higher-grade rectal cancers. ${ }^{109}$

Since the R0 resection of liver metastases from colorectal cancer is the main determinant of long-term disease-free and overall survival, there is importance in determining the response of metastases to therapy. Due to variability in timing and modality of treatment, these patients can often be difficult to standardize. Chan et al examined 45 patients with hepatic-only colorectal metastases who underwent sandwich chemotherapy and then resection. If there was $<10 \%$ viable tumor cells in all resected lesions (which was $34 \%$ of their patient population), there was a statistically significant longer overall survival ( $p$ 0.019). Pathologic graded response was also found to be an independent predictor of survival. ${ }^{110}$ 


\section{Conclusion}

The monitoring of colorectal metastases has undergone very few changes in the last ten years, though, with improvements in systemic therapy, liver-directed therapy, and with our understanding of how to treat the primary, survival may well continue to increase. On the other hand, changes in the way clinicians treat rectal cancer has led to a new age of therapy guidance by imaging and pathology. There are more potential paradigm shifts on the horizon for the treatment of rectal cancer. With the ability to predict complete responders before excision, and good data showing comparable long-term, disease-free, and overall survival, we may see an evolution toward elimination of radical surgery in all except for those who demonstrate local recurrence after neoadjuvant therapy. This, as well as optimal management of patients presenting with stage IV colon cancer, will require precise and accurate modalities to monitor treatment response.

\section{Disclaimers}

The views expressed are those of the author(s) and do not reflect the official policy of the Department of the Army, the Department of Defense or the U.S. Government.

\section{Competing Interests}

The authors have declared that no competing interest exists.

\section{References}

1. Howlader N NA, Krapcho M, Neyman N, Aminou R, Waldron W, Altekruse SF, Kosary CL, Ruhl J, Tatalovich Z, Cho H, Mariotto A, Eisner MP, Lewis DR, Chen HS, Feuer EJ, Cronin KA. SEER Cancer Statistics Review, 1975-2009 (Vintage 2009 Populations). National Cancer Institute. 2012.

2. Welch JP, Donaldson GA. The clinical correlation of an autopsy study of recurrent colorectal cancer. Annals of surgery. 1979;189(4):496-502

3. Borner MM. Neoadjuvant chemotherapy for unresectable liver metastases of colorectal cancer--too good to be true? Annals of oncology : official journal of the European Society for Medical Oncology / ESMO. 1999;10(6):623-626.

4. Lawes D, Boulos PB. Advances in the management of rectal cancer. Journal of the Royal Society of Medicine. 2002;95(12):587-590.

5. Janjan NA, Crane C, Feig BW, et al. Improved overall survival among responders to preoperative chemoradiation for locally advanced rectal cancer. American journal of clinical oncology. 2001;24(2):107-112.

6. Cukier M, Smith AJ, Milot L, et al. Neoadjuvant chemoradiotherapy and multivisceral resection for primary locally advanced adherent colon cancer: a single institution experience. European journal of surgical oncology : the journal of the European Society of Surgical Oncology and the British Association of Surgical Oncology. 2012;38(8):677-682.

7. Huh JW, Kim HR, Kim YJ. Clinical prediction of pathological complete response after preoperative chemoradiotherapy for rectal cancer. Diseases of the colon and rectum. 2013;56(6):698-703.

8. Beck DE. American Society of Colon and Rectal Surgeons. The ASCRS manual of colon and rectal surgery. New York, NY: Springer; 2009.

9. Kosinski L, Habr-Gama A, Ludwig K, Perez R. Shifting concepts in rectal cancer management: a review of contemporary primary rectal cancer treatment strategies. CA: a cancer journal for clinicians. 2012;62(3):173-202.

10. Milsom JW, Bohm B, Hammerhofer KA, Fazio V, Steiger E, Elson P. A prospective, randomized trial comparing laparoscopic versus conventional techniques in colorectal cancer surgery: a preliminary report. Journal of the American College of Surgeons. 1998;187(1):46-54; discussion 54-45.

11. Tournigand C, Andre T, Achille E, et al. FOLFIRI followed by FOLFOX6 or the reverse sequence in advanced colorectal cancer: a randomized GERCOR study. Journal of clinical oncology : official journal of the American Society of Clinical Oncology.2004;22(2):229-237.
12. Jemal A, Siegel R, Ward E, Hao Y, Xu J, Thun MJ. Cancer statistics, 2009. CA: a cancer journal for clinicians. 2009;59(4):225-249.

13. Goldberg RM, Sargent DJ, Morton RF, et al. A randomized controlled trial of fluorouracil plus leucovorin, irinotecan, and oxaliplatin combinations in patients with previously untreated metastatic colorectal cancer. Journal of clinical oncology : official journal of the American Society of Clinical Oncology. 2004;22(1):23-30.

14. Marin JJ, Sanchez de Medina F, Castano B, et al. Chemoprevention, chemotherapy, and chemoresistance in colorectal cancer. Drug metabolism reviews. 2012;44(2):148-172.

15. Hurwitz H, Fehrenbacher L, Novotny W, et al. Bevacizumab plus irinotecan, fluorouracil, and leucovorin for metastatic colorectal cancer. The New England journal of medicine. Jun 3 2004;350(23):2335-2342.

16. Ahmed SL, et al. Survival impact of surgical resection of primary tumor (SRPT) in metastatic colorectal cancer (mCRC): A population-based cohort study. J Clin Oncol 2012; 30: (suppl 34): abstr456.

17. Fahrni AMR. Effect of primary tumor resection on survival in patients with metastatic colorectal cancer in the post-2000 era: A California Cancer Registry analysis. J Clin Oncol 2012; 30: (suppl 34): abstr504.

18. Gresham GR, et al. The impact of palliative resection of the primary tumor on overall survival in metastatic colorectal cancer. 2013.

19. McCahill LY, et al. A phase II trial of 5-fluorouracil, leucovorin, and oxaliplatin (mFOLFOX6) chemotherapy plus bevacizumab for patients with unresectable stage IV colon cancer and a synchronous asymptomatic primary tumor: Updated results of NSABP C-10 with definitive survival analysis. 2013.

20. Wong SF, et al. Metastatic colorectal cancer (mCRC) with primary in situ: An Australian Registry. 2013.

21. Poston GJ, Adam R, Alberts S, et al. OncoSurge: a strategy for improving resectability with curative intent in metastatic colorectal cancer. Journal of clinical oncology : official journal of the American Society of Clinical Oncology. 2005;23(28):7125-7134.

22. Haddad AJ, Bani Hani M, Pawlik TM, Cunningham SC. Colorectal liver metastases. International journal of surgical oncology. 2011;2011:285840.

23. Holleb AI. William Ernest Miles (1869-1947). CA: a cancer journal for clinicians. 1971;21(6):360.

24. Allal AS, Bieri S, Pelloni A, et al. Sphincter-sparing surgery after preoperative radiotherapy for low rectal cancers: feasibility, oncologic results and quality of life outcomes. British journal of cancer. 2000;82(6):1131-1137.

25. Gerard A, Buyse M, Nordlinger B, et al. Preoperative radiotherapy as adjuvant treatment in rectal cancer. Final results of a randomized study of the European Organization for Research and Treatment of Cancer (EORTC). Annals of surgery. 1988;208(5):606-614

26. Rider WD, Palmer JA, Mahoney LJ, Robertson CT. Preoperative irradiation in operable cancer of the rectum: report of the Toronto trial. Canadian journal of surgery. Journal canadien de chirurgie. 1977;20(4):335-338.

27. [No authors listed]. The evaluation of low dose pre-operative X-ray therapy in the management of operable rectal cancer; results of a randomly controlled trial. The British journal of surgery. 1984;71(1):21-25.

28. Improved survival with preoperative radiotherapy in resectable rectal cancer. Swedish Rectal Cancer Trial. The New England journal of medicine. 1997;336(14):980-987.

29. Francois Y, Nemoz CJ, Baulieux J, et al. Influence of the interval between preoperative radiation therapy and surgery on downstaging and on the rate of sphincter-sparing surgery for rectal cancer: the Lyon R90-01 randomized trial. Journal of clinical oncology : official journal of the American Society of Clinical Oncology. 1999;17(8):2396.

30. Gambacorta MA, Valentini V, Morganti AG, et al. Chemoradiation with raltitrexed (Tomudex) in preoperative treatment of stage II-III resectable rectal cancer: a phase II study. International journal of radiation oncology, biology, physics. 2004;60(1):130-138

31. Grann A, Feng C, Wong D, et al. Preoperative combined modality therapy for clinically resectable uT3 rectal adenocarcinoma. International journal of radiation oncology, biology, physics. 2001;49(4):987-995.

32. Rengan R, Paty P, Wong WD, et al. Distal cT2N0 rectal cancer: is there an alternative to abdominoperineal resection? Journal of clinical oncology : official journal of the American Society of Clinical Oncology. 2005;23(22):4905-4912.

33. Sauer R, Becker H, Hohenberger W, et al. Preoperative versus postoperative chemoradiotherapy for rectal cancer. The New England journal of medicine. 2004;351(17):1731-1740

34. Sauer R, Liersch T, Merkel S, et al. Preoperative versus postoperative chemoradiotherapy for locally advanced rectal cancer: results of the German CAO/ARO/AIO-94 randomized phase III trial after a median follow-up of 11 years. Journal of clinical oncology : official journal of the American Society of Clinical Oncology. 2012;30(16):1926-1933.

35. Valentini V, Coco C, Cellini N, et al. Preoperative chemoradiation for extraperitoneal T3 rectal cancer: acute toxicity, tumor response, and sphincter preservation. International journal of radiation oncology, biology, physics. 1998:40(5):1067-1075.

36. Group MS. Diagnostic accuracy of preoperative magnetic resonance imaging in predicting curative resection of rectal cancer: prospective observational study. Bmj. 2006;333(7572):779.

37. Brown G, Davies S, Williams GT, et al. Effectiveness of preoperative staging in rectal cancer: digital rectal examination, endoluminal ultrasound or magnetic resonance imaging? British journal of cancer. 2004;91(1):23-29. 
38. Guillem JG, Chessin DB, Shia J, et al. Clinical examination following preoperative chemoradiation for rectal cancer is not a reliable surrogate end point. Journal of clinical oncology : official journal of the American Society of Clinical Oncology. 2005;23(15):3475-3479.

39. Kirat HT, Ozturk E, Lavery IC, Kiran RP. The predictive value of preoperative carcinoembryonic antigen level in the prognosis of colon cancer. American journal of surgery. 2012;204(4):447-452.

40. Fletcher RH. Carcinoembryonic antigen. Annals of internal medicine. 1986;104(1):66-73.

41. Jang NY, Kang SB, Kim DW, et al. The role of carcinoembryonic antigen after neoadjuvant chemoradiotherapy in patients with rectal cancer. Diseases of the colon and rectum. 2011;54(2):245-252.

42. Sturgeon CM, Duffy MJ, Stenman UH, et al. National Academy of Clinical Biochemistry laboratory medicine practice guidelines for use of tumor markers in testicular, prostate, colorectal, breast, and ovarian cancers. Clinical chemistry. 2008;54(12):e11-79.

43. Perez RO, Sao Juliao GP, Habr-Gama A, et al. The role of carcinoembriogenic antigen in predicting response and survival to neoadjuvant chemoradiotherapy for distal rectal cancer. Diseases of the colon and rectum. 2009;52(6):1137-1143.

44. Willkomm P, Bender H, Bangard M, Decker P, Grunwald F, Biersack HI. FDG PET and immunoscintigraphy with $99 \mathrm{mTc}$-labeled antibody fragments for detection of the recurrence of colorectal carcinoma. Journal of nuclear medicine : official publication, Society of Nuclear Medicine. 2000;41(10):1657-1663.

45. Libutti SK, Alexander HR, Jr., Choyke P, et al. A prospective study of 2-[18F] fluoro-2-deoxy-D-glucose/positron emission tomography scan, 99mTc-labeled arcitumomab (CEA-scan), and blind second-look laparotomy for detecting colon cancer recurrence in patients with increasing carcinoembryonic antigen levels. Annals of surgical oncology. 2001;8(10):779-786.

46. Cohen AM, Martin EW, Jr., Lavery I, et al. Radioimmunoguided surgery using iodine 125 B72.3 in patients with colorectal cancer. Archives of surgery. 1991;126(3):349-352.

47. Kim JC, Kim WS, Ryu JS, et al. Applicability of carcinoembryonic antigen-specific monoclonal antibodies to radioimmunoguided surgery for human colorectal carcinoma. Cancer research. 2000;60(17):4825-4829.

48. Zitt M, Zitt M, Muller HM. DNA methylation in colorectal cancer--impact on screening and therapy monitoring modalities? Disease markers. 2007;23(1-2):51-71.

49. Ahn JB, Chung WB, Maeda O, et al. DNA methylation predicts recurrence from resected stage III proximal colon cancer. Cancer. 2011;117(9):1847-1854.

50. Soldevilla B, Diaz R, Silva J, et al. Prognostic impact of DeltaTAp73 isoform levels and their target genes in colon cancer patients. Clinical cancer research : an official journal of the American Association for Cancer Research. 2011;17(18):6029-6039.

51. Negin BP, Cohen SJ. Circulating tumor cells in colorectal cancer: past, present, and future challenges. Current treatment options in oncology. 2010;11(1-2):1-13.

52. Lu CY, Uen YH, Tsai HL, et al. Molecular detection of persistent postoperative circulating tumour cells in stages II and III colon cancer patients via multiple blood sampling: prognostic significance of detection for early relapse. British journal of cancer. 2011;104(7):1178-1184.

53. Popat S, Matakidou A, Houlston RS. Thymidylate synthase expression and prognosis in colorectal cancer: a systematic review and meta-analysis. Journal of clinical oncology : official journal of the American Society of Clinical Oncology. 2004;22(3):529-536.

54. Bipat S, Glas AS, Slors FJ, Zwinderman AH, Bossuyt PM, Stoker J. Rectal cancer: local staging and assessment of lymph node involvement with endoluminal US, CT, and MR imaging--a meta-analysis. Radiology. 2004;232(3):773-783.

55. Pettersson D, Cedermark B, Holm T, et al. Interim analysis of the Stockholm III trial of preoperative radiotherapy regimens for rectal cancer. The British journal of surgery. 2010;97(4):580-587.

56. Kerr SF, Norton S, Glynne-Jones R. Delaying surgery after neoadjuvant chemoradiotherapy for rectal cancer may reduce postoperative morbidity without compromising prognosis. The British journal of surgery. 2008;95(12):1534-1540.

57. Glehen O, Chapet O, Adham M, Nemoz JC, Gerard JP, Lyons Oncology G. Long-term results of the Lyons $\mathrm{R} 90-01$ randomized trial of preoperative radiotherapy with delayed surgery and its effect on sphincter-saving surgery in rectal cancer. The British journal of surgery. 2003;90(8):996-998.

58. Valentini V, Glimelius B. Rectal cancer radiotherapy: towards European consensus. Acta oncologica. 2010;49(8):1206-1216.

59. Habr-Gama A, Perez RO, Nadalin W, et al. Long-term results of preoperative chemoradiation for distal rectal cancer correlation between final stage and survival. Journal of gastrointestinal surgery : official journal of the Society for Surgery of the Alimentary Tract. 2005;9(1):90-99; discussion 99-101.

60. Lahaye MJ, Beets GL, Engelen SM, et al. Locally advanced rectal cancer: MR imaging for restaging after neoadjuvant radiation therapy with concomitant chemotherapy. Part II. What are the criteria to predict involved lymph nodes? Radiology. 2009;252(1):81-91.

61. Torabi M, Aquino SL, Harisinghani MG. Current concepts in lymph node imaging. Journal of nuclear medicine : official publication, Society of Nuclear Medicine. 2004;45(9):1509-1518.
62. Brown G, Richards CJ, Bourne MW, et al. Morphologic predictors of lymph node status in rectal cancer with use of high-spatial-resolution MR imaging with histopathologic comparison. Radiology. 2003;227(2):371-377.

63. Kwok H, Bissett IP, Hill GL. Preoperative staging of rectal cancer. International journal of colorectal disease. 2000;15(1):9-20.

64. Guillem JG, Ruby JA, Leibold T, et al. Neither FDG-PET Nor CT Is Able to Distinguish Between a Pathological Complete Response and an Incomplete Response After Neoadjuvant Chemoradiation in Locally Advanced Rectal Cancer: A Prospective Study. Annals of surgery. 2012.

65. Therasse P, Arbuck SG, Eisenhauer EA, et al. New guidelines to evaluate the response to treatment in solid tumors. European Organization for Research and Treatment of Cancer, National Cancer Institute of the United States, National Cancer Institute of Canada. Journal of the National Cancer Institute. 2000;92(3):205-216.

66. Monteil J, Mahmoudi N, Leobon S, et al. Chemotherapy response evaluation in metastatic colorectal cancer with FDG PET/CT and CT scans. Anticancer research. 2009;29(7):2563-2568.

67. Jaffe CC. Measures of response: RECIST, WHO, and new alternatives. Journal of clinical oncology : official journal of the American Society of Clinical Oncology. 2006;24(20):3245-3251.

68. Huebner RH, Park KC, Shepherd JE, et al. A meta-analysis of the literature for whole-body FDG PET detection of recurrent colorectal cancer. Journal of nuclear medicine : official publication, Society of Nuclear Medicine. 2000;41(7):1177-1189.

69. Brush J, Boyd K, Chappell F, et al. The value of FDG positron emission tomography/computerised tomography (PET/CT) in pre-operative staging of colorectal cancer: a systematic review and economic evaluation. Health technology assessment. 2011;15(35):1-192.

70. Dobos N, Rubesin SE. Radiologic imaging modalities in the diagnosis and management of colorectal cancer. Hematology/oncology clinics of North America. 2002;16(4):875-895.

71. National Collaborating Centre for Cancer (UK). Colorectal Cancer: The Diagnosis and Management of Colorectal Cancer. Cardiff: National Collaborating Centre for Cancer (UK). 2011.

72. Conti PS, Lilien DL, Hawley K, Keppler J, Grafton ST, Bading JR. PET and $[18 \mathrm{~F}]-\mathrm{FDG}$ in oncology: a clinical update. Nuclear medicine and biology. 1996;23(6):717-735.

73. Delbeke D. Oncological applications of FDG PET imaging: brain tumors, colorectal cancer, lymphoma and melanoma. Journal of nuclear medicine : official publication, Society of Nuclear Medicine. 1999;40(4):591-603.

74. Delbeke D, Vitola JV, Sandler MP, et al. Staging recurrent metastatic colorectal carcinoma with PET. Journal of nuclear medicine : official publication, Society of Nuclear Medicine. 1997;38(8):1196-1201

75. Flanagan FL, Dehdashti F, Ogunbiyi OA, Kodner IJ, Siegel BA. Utility of FDG-PET for investigating unexplained plasma CEA elevation in patients with colorectal cancer. Annals of surgery. 1998;227(3):319-323.

76. Lai DT, Fulham M, Stephen MS, et al. The role of whole-body positron emission tomography with [18F]fluorodeoxyglucose in identifying operable colorectal cancer metastases to the liver. Archives of surgery. 1996;131(7):703-707.

77. Capirci C, Rubello D, Chierichetti F, et al. Restaging after neoadjuvant chemoradiotherapy for rectal adenocarcinoma: role of F18-FDG PET. Biomedicine \& pharmacotherapy $=$ Biomedecine \& pharmacotherapie. 2004;58(8):451-457.

78. Kristiansen C, Loft A, Berthelsen AK, et al. PET/CT and histopathologic response to preoperative chemoradiation therapy in locally advanced rectal cancer. Diseases of the colon and rectum. 2008;51(1):21-25.

79. Denecke T, Rau B, Hoffmann KT, et al. Comparison of CT, MRI and FDG-PET in response prediction of patients with locally advanced rectal cancer after multimodal preoperative therapy: is there a benefit in using functional imaging? European radiology. 2005;15(8):1658-1666

80. O'Neill BD, Brown G, Heald RJ, Cunningham D, Tait DM. Non-operative treatment after neoadjuvant chemoradiotherapy for rectal cancer. The lancet oncology. 2007;8(7):625-633.

81. Dewhurst CE, Mortele KJ. Magnetic resonance imaging of rectal cancer. Radiologic clinics of North America. 2013;51(1):121-131.

82. Brown G, Radcliffe AG, Newcombe RG, Dallimore NS, Bourne MW, Williams GT. Preoperative assessment of prognostic factors in rectal cancer using high-resolution magnetic resonance imaging. The British journal of surgery. 2003;90(3):355-364.

83. Patel UB, Blomqvist LK, Taylor F, et al. MRI after treatment of locally advanced rectal cancer: how to report tumor response--the MERCURY experience. AJR. American journal of roentgenology. 2012;199(4):W486-495.

84. Kim NK, Baik SH, Min BS, et al. A comparative study of volumetric analysis, histopathologic downstaging, and tumor regression grade in evaluating tumor response in locally advanced rectal cancer following preoperative chemoradiation. International journal of radiation oncology, biology, physics. 2007;67(1):204-210

85. Kang JH, Kim YC, Kim H, et al. Tumor volume changes assessed by three-dimensional magnetic resonance volumetry in rectal cancer patients after preoperative chemoradiation: the impact of the volume reduction ratio on the prediction of pathologic complete response. International journal of radiation oncology, biology, physics. 2010;76(4):1018-1025.

86. Kluza E, Rozeboom ED, Maas M, et al. T2 weighted signal intensity evolution may predict pathological complete response after treatment for rectal cancer. European radiology. 2013;23(1):253-261. 
87. Kuo LJ, Chiou JF, Tai CJ, et al. Can we predict pathologic complete response before surgery for locally advanced rectal cancer treated with preoperative chemoradiation therapy? International journal of colorectal disease. 2012;27(5):613-621.

88. Edelman BR, Weiser MR. Endorectal ultrasound: its role in the diagnosis and treatment of rectal cancer. Clinics in colon and rectal surgery. 2008;21(3):167-177.

89. Pastor C, Subtil JC, Sola J, et al. Accuracy of endoscopic ultrasound to assess tumor response after neoadjuvant treatment in rectal cancer: can we trust the findings? Diseases of the colon and rectum. 2011;54(9):1141-1146.

90. Kav T, Bayraktar Y. How useful is rectal endosonography in the staging of rectal cancer? World journal of gastroenterology : WJG. 2010;16(6):691-697.

91. Fernandez-Esparrach G, Ayuso-Colella JR, Sendino O, et al. EUS and magnetic resonance imaging in the staging of rectal cancer: a prospective and comparative study. Gastrointestinal endoscopy. 2011;74(2):347-354.

92. Maor Y, Nadler M, Barshack I, et al. Endoscopic ultrasound staging of rectal cancer: diagnostic value before and following chemoradiation. Journal of gastroenterology and hepatology. 2006;21(2):454-458.

93. Napoleon B, Pujol B, Berger F, Valette PJ, Gerard JP, Souquet JC. Accuracy of endosonography in the staging of rectal cancer treated by radiotherapy. The British journal of surgery. 1991;78(7):785-788.

94. Assenat E, Thezenas S, Samalin E, et al. The value of endoscopic rectal ultrasound in predicting the lateral clearance and outcome in patients with lower-third rectal adenocarcinoma. Endoscopy. 2007;39(4):309-313.

95. Gavioli M, Bagni A, Piccagli I, Fundaro S, Natalini G. Usefulness of endorectal ultrasound after preoperative radiotherapy in rectal cancer: comparison between sonographic and histopathologic changes. Diseases of the colon and rectum. 2000;43(8):1075-1083.

96. Rovera F, Dionigi G, Boni L, Cutaia S, Diurni M, Dionigi R. The role of EUS and MRI in rectal cancer staging. Surgical oncology. 2007;16 Suppl 1:S51-52.

97. Hiotis SP, Weber SM, Cohen AM, et al. Assessing the predictive value of clinical complete response to neoadjuvant therapy for rectal cancer: an analysis of 488 patients. Journal of the American College of Surgeons. 2002;194(2):131-135; discussion 135-136.

98. Glynne-Jones R, Wallace M, Livingstone JI, Meyrick-Thomas J. Complete clinical response after preoperative chemoradiation in rectal cancer: is a "wait and see" policy justified? Diseases of the colon and rectum. 2008;51(1):10-19; discussion 19-20.

99. Maas M, Beets-Tan RG, Lambregts DM, et al. Wait-and-see policy for clinical complete responders after chemoradiation for rectal cancer. Journal of clinical oncology : official journal of the American Society of Clinical Oncology. 2011;29(35):4633-4640.

100. Smith JD, Ruby JA, Goodman KA, et al. Nonoperative management of rectal cancer with complete clinical response after neoadjuvant therapy. Annals of surgery. 2012;256(6):965-972.

101. Dworak O, Keilholz L, Hoffmann A. Pathological features of rectal cancer after preoperative radiochemotherapy. International journal of colorectal disease. 1997;12(1):19-23.

102. MacGregor TP, Maughan TS, Sharma RA. Pathological grading of regression following neoadjuvant chemoradiation therapy: the clinical need is now. Journal of clinical pathology. 2012;65(10):867-871.

103. Washington MK, Berlin J, Branton P, et al. Protocol for the examination of specimens from patients with primary carcinoma of the colon and rectum. Archives of pathology \& laboratory medicine. 2009;133(10):1539-1551.

104. Park YJ, Oh BR, Lim SW, et al. Clinical significance of tumor regression grade in rectal cancer with preoperative chemoradiotherapy. Journal of the Korean Society of Coloproctology. 2010;26(4):279-286.

105. Rodel C, Martus P, Papadoupolos T, et al. Prognostic significance of tumor regression after preoperative chemoradiotherapy for rectal cancer. Journal of clinical oncology : official journal of the American Society of Clinical Oncology. 2005;23(34):8688-8696.

106. Martin ST, Heneghan HM, Winter DC. Systematic review and meta-analysis of outcomes following pathological complete response to neoadjuvant chemoradiotherapy for rectal cancer. The British journal of surgery. 2012;99(7):918-928.

107. Maas M, Nelemans PJ, Valentini V, et al. Long-term outcome in patients with a pathological complete response after chemoradiation for rectal cancer: a pooled analysis of individual patient data. The lancet oncology. 2010;11(9):835-844.

108. Matrai Z, Lovey J, Hitre E, et al. [Histologic response after neoadjuvant therapy in rectal adenocarcinoma: own experience and review of the literature]. Orvosi hetilap. 22 2006;147(42):2011-2020.

109. Hughes R, Glynne-Jones R, Grainger J, et al. Can pathological complete response in the primary tumour following pre-operative pelvic chemoradiotherapy for T3-T4 rectal cancer predict for sterilisation of pelvic lymph nodes, a low risk of local recurrence and the appropriateness of local excision? International journal of colorectal disease. 2006;21(1):11-17.

110. Chan G, Hassanain M, Chaudhury $P$, et al. Pathological response grade of colorectal liver metastases treated with neoadjuvant chemotherapy. HPB : the official journal of the International Hepato Pancreato Biliary Association. 2010;12(4):277-284.

111. Chennupati SK, Quon A, Kamaya A, et al. Positron emission tomography for predicting pathologic response after neoadjuvant chemoradiotherapy for locally advanced rectal cancer. American journal of clinical oncology. 2012;35(4):334-339.
112. Group MS. Extramural depth of tumor invasion at thin-section MR in patients with rectal cancer: results of the MERCURY study. Radiology. 2007;243(1):132-139.

113. Wieder HA, Geinitz $H$, Rosenberg $R$, et al. PET imaging with $[18 \mathrm{~F}] 3$ '-deoxy-3'-fluorothymidine for prediction of response to neoadjuvant treatment in patients with rectal cancer. European journal of nuclear medicine and molecular imaging. 2007;34(6):878-883.

114. Habr-Gama A, Perez RO, Nadalin W, et al. Operative versus nonoperative treatment for stage 0 distal rectal cancer following chemoradiation therapy: long-term results. Annals of surgery. 2004;240(4):711-717; discussion 717-718.

115. Maretto I, Pomerri F, Pucciarelli S, et al. The potential of restaging in the prediction of pathologic response after preoperative chemoradiotherapy for rectal cancer. Annals of surgical oncology. 2007;14(2):455-461.

116. Intven M, Reerink O, Philippens ME. Diffusion-weighted MRI in locally advanced rectal cancer : pathological response prediction after neo-adjuvant radiochemotherapy. Strahlentherapie und Onkologie: Organ der Deutschen Rontgengesellschaft. 2013;189(2):117-122. 